\title{
Repeater for 5G Wireless: A Complementary Contender for Spectrum Sensing Intelligence
}

\author{
Shree Krishna Sharma*, Mohammad Patwary ${ }^{\dagger}$, Symeon Chatzinotas*, Björn Ottersten*, Mohamed Abdel-Maguid ${ }^{\ddagger}$ \\ ${ }^{*}$ SnT - securityandtrust.lu, University of Luxembourg, Luxembourg \\ Email: \{shree.sharma, symeon.chatzinotas, bjorn.ottersten\}@uni.lu \\ $\dagger$ FCES, Staffordshire University, United Kingdom, Email: M.N.Patwary@staffs.ac.uk \\ ‡ University Campus Suffolk, United Kingdom, Email: m.maguid@ucs.ac.uk
}

\begin{abstract}
Exploring innovative cellular architectures to achieve enhanced system capacity and good coverage has become a critical issue towards realizing the fifth generation (5G) of wireless communications. In this context, this paper proposes a novel concept of an intelligent Amplify and Forward (AF) 5G repeater for enabling the densification of future cellular networks. The proposed repeater features a Spectrum Sensing (SS) intelligence capability and utilizes such intelligence in a complementary fashion in comparison to its existing counterpart (e.g., Cognitive Radio) by detecting the active channels within the assigned spectrum. This intelligence allows the proposed repeater to carry out selective amplification of the active channels in contrast to the full amplification in conventional AF repeaters. Furthermore, the performance of a Frequency Division Multiple Access (FDMA) based two hop cellular network utilizing the proposed repeater is evaluated in terms of the system throughput. Simulation results demonstrate up to $13 \%$ increase when compared with the conventional repeaters. Moreover, the effect of SS errors on the system capacity is analyzed.
\end{abstract}

\section{INTRODUCTION}

The fifth generation $(5 \mathrm{G})$ of wireless communications is targeting enhanced data rates and better quality of service with proper fairness among multiple users [1]. Although current Long Term Evolution-Advanced (LTE-A) technology can provide high data rate access on the air interface, providing higher capacity to the areas having high user density with diverse traffic types and data rate demand is still challenging. Further, in urban areas, the call dropping problem becomes more annoying to the customers than the call blocking problem [2]. One of the cost-effective solutions to address the aforementioned issue is to deploy repeaters in specific urban areas where severe call dropping has been reported based on network utilization reports. Additionally, in rural and sub-urban areas, the deployment of repeaters significantly helps in extending the coverage area of a cellular system [3].

In today's wireless networks, the statement that "repeaters are for coverage enhancement only" has become a misconception [4]. With the advancement of technology, the system capacity gain that can be obtained from the repeaters has become more significant [2]. In this context, several contributions have investigated the application of repeaters in different environments [2], [5], [6]. One of the aims of deploying repeaters is to enhance the Signal to Interference plus Noise Ratio (SINR) of cell-edge users, which is equivalent to bring users from the cell-edge much closer to the cell center. This increased SINR directly translates into an increased data rate, thus the deployment of a repeater can significantly enhance the network capacity [4]. In $4 \mathrm{G}$ and beyond $4 \mathrm{G}$ networks, the data service is the main priority and the system capacity is mainly measured as the aggregate sector throughput which can be contributed from every parts of the network regardless of their locations. In addition to the capacity enhancement due to increased Modulation and Coding Scheme (MCS) and improved service time allocation, repeaters can enhance the Multiple Input Multiple Output (MIMO) gain under Line of Sight (LoS) conditions. In this context, the Third Generation Partnership Project (3GPP) has already identified relaying as a potential technique in order to increase the coverage as well as to enhance the system throughput [7].

Related Literature: In the existing multihop cellular literature, there exist the following two categories of devices for enhancing the signal levels [8]: (i) Layer 1 repeater, and (ii) Layer 2 relay. Layer 2 relays basically operate in a Decode and Forward (DF) mode whereas layer 1 repeaters work in an Amplify and forward (AF) mode. The DF relays can provide better noise immunity and better intercell interference avoidance/mtigation than AF repeaters. However, the DF relay requires a complicated transceiver and can further introduce the transmission delay. One of the main aims of $5 \mathrm{G}$ networks is to reduce the data transmission delay present in the current wireless networks. In this context, it is crucial to explore the applications of AF repeaters in comparison to DF relays for delay sensitive services. For example, the additional delay caused by the layer 2 relay may be unacceptable for uplink signalling in IEEE 802.16 based systems [9]. Herein, the AF repeater is of practical interest and hence investigated in this paper since it simply applies a linear signal processing operation on the signals and has low implementation complexity [10].

Several contributions in the literature have studied various spectrum awareness techniques such as Spectrum Sensing (SS), Signal to Noise Ratio (SNR) and sparsity order estimation in the context of a Cognitive Radio (CR) [11]-[14]. Further, cognitive relay networks have been investigated in several contributions mainly focusing on the DF relay scenario [6], [15], [16]. Recently, the contribution in [17] investigates the performance of cognitive AF repeaters in a dense urban environment. It has been demonstrated that the AF repeater can be used in LTE-A based systems in order to offload mobile traffic within unused frequency bands effectively. Further, authors in [18] have studied the deployment of a cognitive relay for indoor scenarios as an underlay system. In addition, authors in [19] propose to deploy several relay stations equipped with a $\mathrm{CR}$ in order to mitigate intercell interference in cellular 
systems. However, the aspect of introducing intelligence in the AF repeater has received limited attention despite its several important aspects and is the main focus of this paper.

Motivation and Contributions: It is well understood in the literature that repeaters can significantly boost the system capacity and increase the coverage area [2], [5], [20]. Despite several advantages of AF repeaters, there exist some disadvantages which limit their practical applicability. The biggest challenge with the AF repeaters is that it will amplify interference as well as noise in unnecessary bands. To address this issue, we propose to have SS intelligence at the repeater which is widely investigated in the context of CR communications [12], [21]. In the CR paradigm, the main aim of SS is to find the spectral holes dynamically in different domains in such a way that they can be allocated to potential Secondary Users (SUs) in order to maximize the spectral efficiency. Motivated by this dynamic SS concept, if we can embed SS intelligence into the repeaters, only necessary bands will be amplified and the interference plus noise amplification problem can be drastically minimized. Hence, there exists a scope of utilization of SS intelligence within the repeater. In contrast to the CR context, the proposed repeaters employ SS intelligence in order to find the spectral peaks (above a certain energy threshold) instead of the spectral holes. Thus the proposed repeater is herein treated as a "complementary contender" for finding out active channels within the assigned spectrum. In future $5 \mathrm{G}$ wireless systems, the proposed repeaters will perform selective amplification of the active channels and hence do not necessarily have to amplify all the other bands, hence saving resources and also reducing the effect of interference and noise.

In this paper, first, we describe the important aspects and system assumptions of the proposed $5 \mathrm{G}$ repeater. Subsequently, we evaluate and compare the system capacity of a cellular system with the proposed repeater than that of the system with the conventional repeater which does not employ SS intelligence. Furthermore, the effect of sensing errors on the system capacity is analyzed with the help of theoretical and numerical analysis.

Organization: The remainder of this paper is organized as follows: Section II proposes the concept of the proposed $5 \mathrm{G}$ repeater along with a practical use case. Section III presents the theoretical analysis for evaluating the cellular system capacity considering the proposed repeater and further studies the effect of SS errors. Section IV evaluates the performance of the proposed approach with the help of numerical results. Finally, Section V concludes the paper.

\section{Proposed 5G RepeAter}

We consider the proposed repeater to be capable of sensing the activity of the channels within the assigned spectrum within a predefined area of interest. From the spectral measurements carried out throughout different parts of the world, it has been widely observed that all the designated channels in a certain cell are often not occupied at the same time and there exist spectrum holes in the temporal as well as in the spatial domains [22]. In this context, it is sufficient for the repeaters to amplify only the active channels at a certain time. However, the traditional repeaters amplify the entire downlink/uplink system bandwidth.
The conventional belief about the repeaters is that they are responsible for amplifying the unnecessary spectrum of information, injecting additional interference to the received signal from the post amplification stage. In order to address the aforementioned problems, we propose an intelligent AF repeater which employs a dynamic SS technique. The employed SS technique can vary from a simple Energy Detection (ED) to the eigenvalue based detection depending on the level of allowable complexity and performance level. For the sake of simplicity, we consider an energy detector in this work. By comparing the received energy levels with a predefined threshold, the employed energy detector finds the dynamic spectrum energy peaks (active channels) as well as regular nonzero energy occupancy within the spectrum of interest. Based on the knowledge of active channels, the proposed repeater performs the selective amplification of the channels instead of the whole assigned bandwidth.

Some of the fundamental assumptions of the proposed $5 \mathrm{G}$ repeater are provided below.

1) The repeaters are expected to be used to implement the small (micro/pico/nano) cell vision of future generation of cellular wireless communication networks.

2) We consider that the proposed repeater is a passive network entity although being a part of the network. All radio-resource management functions such as mobility, retransmission mechanism and scheduling are handled by the BS. Although the network can provide with a list of active users within the cell as well as within the network, the repeater with the proposed intelligence will require location specific active user and the SS intelligence within the repeater will allow it to discover such information.

3) The proposed repeater operates in on-frequency mode with sufficient antenna isolation.

4) The repeaters are to be assigned with predefined coverage areas. Beamforming intelligence is also assumed to be incorporated within the repeaters to maintain coverage within the predefined area of interest, thus implementing the Space Division Multiple Access (SDMA) technology.

5) The repeaters can sense active occupancy of the spectrum within the spectrum of interest. For this purpose, an energy threshold is predefined to determine the activity chart of the user group of the interest within the coverage area of interest as well as to obtain neighbourhood user groups. The location of the active users can also be obtained from co-operation among neighbouring repeaters or with the help of source localization techniques within the same repeater.

6) We assume that the BS can be equipped with either omnidirectional/sectorized or beam-steered transmit receive Radio Frequency (RF) front-end. Further, the repeater's donor antenna (accepting from the BS) is to be either omnidirectional or highly directive while the repeater's serving antenna is to be precisely directive to the User Terminal (UT)/specific user groups. The directivity of the repeater's serving beam to the UT is steered by the source localization intelligence while the selectivity of the serving spectrum is governed by the SS intelligence. 


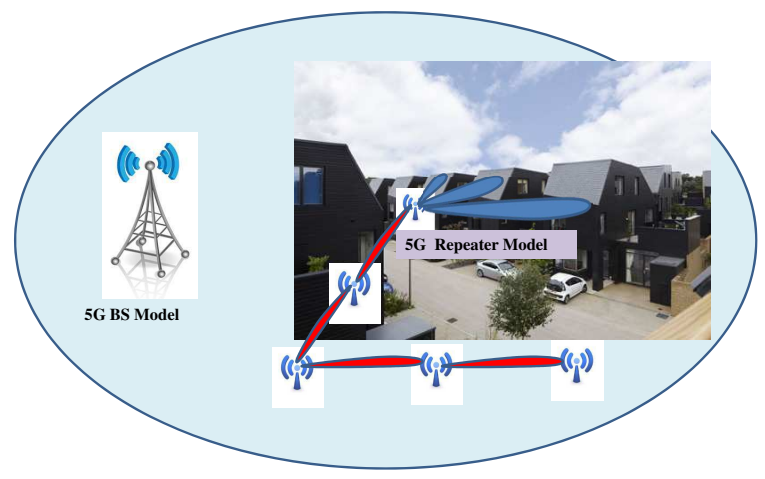

Fig. 1. Use case illustrating the application of the proposed $5 \mathrm{G}$ repeater

7) Further, we assume the repeater to be locally powered.

8) Moreover, at least three neighbouring repeaters can serve or negotiate the serving area (space) in a cooperative manner for implementing SDMA.

We distinguish the proposed repeater from the widely discussed pico BS for future 5G wireless communications in the fact that the repeater does not need additional bandwidth due its on-frequency operation and does not require any backhaul for signalling with the BS. Further, lower output power, smaller size and reduced cost are other distinctive features of a repeater from the traditional BS. It should be noted that implementing SS intelligence within the repeater and other full duplex as well as beamforming capabilities make the repeater more complex than the conventional repeaters. However, it is expected to be cost effective from overall business perspective compared to its counterparts.

The main objectives of the proposed repeater are: (i) to enhance the cellular capacity in the urban areas with high traffic demand, (ii) to extend coverage in rural areas, and (iii) to save transmit power (accumulating from BS and the repeaters) to the UT in order to maintain the link quality. Out of these objectives, we focus on the first objective on this paper.

\section{A. Use Case}

Based on the aforementioned description, we describe the following practical use case of the proposed repeater. An example of the use case is depicted in Fig. 1. We consider that the BS is equipped with an omnidirectional antenna while the serving antenna of the repeater is directive to a user or a specific user group. The UT's receive antenna is assumed to be omnidirectional. There exist the following two links from the BS to the user: (i) direct link from the BS to the user, (ii) the link from the BS to the user via repeater. These two links may increase the diversity for improving the signal quality at the user. Further, BS and repeaters can also serve separate user groups, for example, repeaters serving the edge-users or the users which are in non-line of sight path from the BS. In this scenario, the return link can be either the direct link or via repeater or both for diversity purpose. In this paper, we focus on the downlink of a cellular system with the repeaters. We consider on-frequency repeater and assume that there is sufficient antenna isolation for this purpose.

\section{B. Repeater Operation}

In the following, we describe a general procedure for the operation of the proposed repeater.

\section{Procedure}

1) A new activity within the spectrum of interest is introduced in the following three cases: (i) when a user requests for an operating channel from the Mobile Switching Centre (MSC), (ii) when a user responds to the BS notification about his call from the second party, and (iii) when a user (on call) enters into the region of interest (repeater's coverage).

2) The repeater senses over the whole spectrum assigned to it and finds all the active channels with the help of the employed SS technique.

3) If a channel is found to be active in step (2), this channel is added to the repeaters' task/amplification list for both uplink and downlink.

4) In the downlink transmission by the BS, the repeater only amplifies the active downlink channels identified in step (2) and in the uplink transmission by the UT, the repeater only amplifies the corresponding uplink channels.

5) When the user terminates the call, the respective frequency pair (uplink/downlink) is deleted from the repeater's task (amplification) list.

6) Steps (2) to (5) are repeated over the duration of repeater operation.

\section{SYSTEM MODEL AND ANALYSIS}

We consider a Frequency Division Multiple Access (FDMA) based two hop cellular network with BS-repeater link and the repeater to the user link. The cellular network is represented with hexagonal cells, each of radius $R$ and the frequency reuse factor is $\tau$. We assume that repeaters are placed at a certain distance from the BS to the cell border and $N_{R}$ number of repeaters per cell are considered. In the considered set up, the desired signal is sent from serving $\mathrm{BS}$ to the user and the interference signal comes from other cochannel BSs to the user. It should be noted that the desired signal can also be retransmitted by the repeaters located in adjacent cells. However, we assume that this retransmission is negligible. The received signal at a repeater is amplified along with the thermal noise and is retransmitted to the user. Finally, the received signals at the UT are summed together as multipath signals.

We assume that repeaters coordinate with each other within a cell and they are powered locally. As mentioned earlier, we assume the beamforming intelligence within each repeater and it is capable of performing SDMA to serve the users/user groups in a specific area within a cell. Although the intracell and intercell interferences can be negligible due to the employed SDMA and coordinated transmission in future $5 \mathrm{G}$ communications, for the completeness of the analysis, we consider cochannel interference at the user caused by the first tier of cochannel BSs. Further, we assume that the interference from the repeaters within the cochannel cells is neglected due to their highly directive transmission to the users/user groups. 
We consider a circular approximation of the hexagonal cellular system and place each BS at the centre of the cell having radius $R$. Then we place repeaters at a distance $r$ from the BS with $r<R$ and consider the same amplification gain for all repeaters.

Let $d_{B S, R}$ denotes the distance from the BS to the repeater and $d_{R, U}$ be the distance from the repeater to the user. The path loss of the link between BS and the repeater can be written as [23]

$$
L_{B S, R}=P_{r, R} / P_{t, B S}=\frac{G_{t, B S} G_{r, R} \lambda^{2}}{(4 \pi)^{2} d_{B S, R}^{\alpha}},
$$

where $P_{r, R}$ denotes the received power by the repeater when $P_{t, B S}$ power is transmitted by the BS, $G_{t, B S}$ denotes the gain of the BS transmit antenna, $G_{r, R}$ is the gain of the repeater receive antenna, $\lambda$ denotes the wavelength of the radio wave corresponding to the operating frequency and $\alpha$ denotes the path loss exponent. We assume that $P_{t, B S}$ is same for all the BSs. Similarly, the path loss of the link between repeater and the user can be written as

$$
L_{R, U}=\frac{P_{r, U}}{P_{t, R}}=\frac{G_{t, R} G_{r, U} \lambda^{2}}{(4 \pi)^{2} d_{R, U}^{\alpha}},
$$

where $P_{r, U}$ denotes the received power by the user, $P_{t, R}$ represents the power transmitted by the repeater, $G_{t, R}$ denotes the gain of the repeater transmit antenna in its main lobe, $G_{r, U}$ is the receive gain of the UT antenna. It should be noted that depending on the antenna patterns of the repeater's serving antenna, the UT may also receive the desired BS signal retransmitted by other repeaters within its cell. Herein, we consider the same sidelobe gain ${ }^{1} G_{r, U, s}$ and the corresponding path loss $L_{R, U, s}$, which can be obtained from (2) by replacing $G_{r, U}$ with $G_{r, U, s}$.

In the considered system model, the received power at the UT can be written as

$P_{r, U}=P_{t, B S}\left(R_{G} L_{B S, R} L_{R, U}+\sum_{i=1}^{N_{R}-1} R_{G} L_{B S, R} L_{R i, U, s}+L_{D}\right)$,

where $R_{G}$ denotes the amplification gain of the repeater and $L_{D}$ denotes the link loss for the direct path from the BS to the user. Further, the interference received at the UT can be written as

$$
I_{r, U}=P_{t, B S} \sum_{i=1}^{M_{c}} L_{B S i, U}
$$

where $M_{c}$ is the number of cochannel cells, $L_{B S i, U}$ denotes the path loss of the link between the $i$ th cochannel BS to the user.

The noise power at the UT, denoted by $N_{U}$, is given by

$$
N_{U}=N_{R} R_{G} L_{R, U}+\sum_{i=1}^{N_{R}-1} N_{R} R_{G} L_{R i, U, s}+N_{0},
$$

where $N_{R}=k T_{R} B$ is the noise power at the repeater, with $k$ being Boltzmann's constant, $T_{R}$ being the noise temperature and $B$ being the total amplification bandwidth at the repeater,

\footnotetext{
${ }^{1}$ In practice, this gain depends on the offset angle of the link between user and the repeater from its boresight direction and can be calculated based on the given antenna pattern and the offset angle.
}

$N_{0}=k T_{U} B_{U}$ is the noise power at the UT with $T_{U}$ and $B_{U}$ being the noise temperature and noise bandwidth at the UT.

Combining (3), (4) and (5), the received SINR at the UT when included repeaters can be written as

$$
S I N R=\frac{P_{t, B S}\left(R_{G} L_{B S, R} L_{R, U}+\sum_{i=1}^{N_{R}-1} R_{G} L_{B S, R} L_{R i, U, s}+L_{D}\right)}{P_{t, B S} \sum_{i=1}^{M_{C}} L_{B S i, U}+N_{U}} \text {,6) }
$$

where $N_{U}$ is given by (5). Then the spectral efficiency of a single user link in the considered scenario is given by

$$
S E=\log _{2}\left(1+\frac{P_{r, U}}{I_{r, U}+N_{U}}\right) \text {. }
$$

Subsequently, the system throughput (per cluster system capacity) is given by

$$
R_{U}=\tau N_{c} B_{c} \log _{2}\left(1+\frac{P_{r, U}}{I_{r, U}+N_{U}}\right)
$$

where $N_{c}$ and $B_{c}$ are the number of channels supported by the BS and the bandwidth of a channel, respectively.

It can be noted that the above system throughput is obtained considering the full amplification of the total noise bandwidth at the repeater. However, in the proposed repeater, only the active channels out of the total channels are amplified, hence reducing the noise bandwidth of the repeater, and hence the overall noise power at the UT. Let $B_{a}$ denotes the reduced bandwidth comprised of only active channels and is defined as

$$
B_{a}=\frac{B}{N_{c}} N_{a}
$$

where $N_{c}$ denotes the total number of channels within the considered bandwidth $B$ and $N_{a}$ denotes the number of active channels identified by the repeater. Subsequently, the total reduced noise power at the UT is given by

$$
N_{U 1}=k T_{R} B_{a} R_{G} L_{R, U}+\sum_{i=1}^{N_{R}-1} k T_{R} B_{a} R_{G} L_{R, U, s}+N_{0} .
$$

Therefore, the achievable throughput with SS intelligence embedded in the repeater can be written as

$$
R_{U 1}=\tau N_{c} B_{c} \log _{2}\left(1+\frac{P_{r, U}}{I_{r, U}+N_{U 1}}\right) .
$$

From (8), and (11), it can be noted that the system capacity with the SS intelligence increases than that of the system capacity without SS intelligence in the repeater.

\section{A. Effect of Spectrum Sensing Errors}

For the SS purpose, we use a simple ED technique in which the activity of the channels is determined by comparing the received energy with the noise floor which is calculated based on a suitable noise variance estimation method. Further, we define $P\left(H_{0}\right)$ as the probability of the PU being inactive, and $P\left(H_{1}\right)$ as the probability of the PU being active in such a way that $P\left(H_{0}\right)+P\left(H_{1}\right)=1$. In this work, we assume the perfect knowledge of the noise power at the receiver. Let $T_{s}$ be the sensing time and $N_{s}$ be the number of samples collected within this duration i.e., $N_{s}=\left\lceil T_{s}\right\rceil f_{s}$ with $f_{s}$ being the sampling frequency at the sensing RF chain of the repeater. The test statistic for the ED technique is given by $T=\frac{1}{N} \sum_{n=1}^{N_{s}}|y(n)|^{2}$ 
where $y(n)=x(n)+z(n)$ is the received signal at the repeater, with $x(n)$ being the active signal and $z(n)$ being the Additive White Gaussian Noise (AWGN). Using binary hypothesis testing, the expressions for probability of false alarm $\left(P_{f}\right)$ and probability of detection $\left(P_{d}\right)$ can be computed by [24]; $P_{f}=\operatorname{Pr}\left(T>\lambda \mid H_{0}\right)$, and $P_{d}=\operatorname{Pr}\left(T>\lambda \mid H_{1}\right)$, where $\lambda$ is the sensing threshold.

It should be noted that (9) provides the active bandwidth assuming the perfect sensing information. However, in practice, there may occur the following sensing errors: (i) $P_{f}$ under the $H_{0}$ hypothesis, and (ii) Probability of miss detection $P_{m}=1-P_{d}$ under the $H_{1}$ hypothesis. By considering these sensing errors into account, the active bandwidth in (9) can be modified into the effective active bandwidth $B_{a e}$ as

$$
B_{a e}=\frac{B}{N_{c}} N_{a e}=\frac{B}{N_{c}}\left(N_{a}\left(1-P_{f}\right) P\left(H_{0}\right)+N_{a} P_{d} P\left(H_{1}\right)\right),
$$

with the corresponding effective noise power given by: $N_{0 e}=$ $k T B_{a e}$. Further, considering a constant false alarm rate based ED detector, the expression for $P_{d}$ can be expressed in terms of the target $\bar{P}_{f}$ in the following way [25]

$$
P_{d}=Q\left(\frac{1}{\sqrt{2 \gamma_{\mathrm{R}}+1}}\left(Q^{-1}\left(\bar{P}_{f}\right)-\sqrt{T_{s} f_{s}} \gamma_{\mathrm{R}}\right)\right) .
$$

where $\gamma_{R}$ is the SNR of the active signal, $Q($.$) is the$ complementary distribution function of the standard Gaussian random variable, given by $Q(x)=\frac{1}{\sqrt{2 \pi}} \int_{x}^{\infty} \exp \left(\frac{-t^{2}}{2}\right) d t$. By substituting the value of $P_{d}$ from (13) in (12), the effective active bandwidth $B_{a e}$ can be expressed in terms of the $\bar{P}_{f}$.

\section{NUMERICAL RESUlTS}

In this section, we carry out the performance of the proposed repeater focusing on the SS intelligence. For this purpose, we consider the downlink of a cellular system with the frequency reuse factor of $\tau=3$ operating in the band $2585-2600 \mathrm{MHz}$ (LTE Frequency Division Duplex (FDD) band 16). The BS is assumed to be located at the centre of the cell having radius $R$. Further, 3 similar repeaters (with the same $R_{G}$ ) are placed at a distance of $r$ from the BS with a separation of $120^{\circ}$. The considered simulation parameters are presented in Table I. Uniformly generated random users were generated throughout the cell area and a user within an specified region is served with a designated repeater assigned to that region with its main lobe. Further, the retransmission of signals from the sidelobes of other repeaters within that specific cell is considered assuming the same sidelobe gain as indicated in Table I. The analysis presented in Section III was followed to calculate the SINR at the UT and the system throughput was calculated by averaging over $10^{3}$ Monte Carlo realizations.

Figure 2 depicts the system throughput versus transmit power for two different values of repeater gain i.e., $80 \mathrm{~dB}$ and $100 \mathrm{~dB}$ considering $20 \%$ active channels over the considered spectrum, as an example. From the figure, it can be noted that system throughput increases with the BS transmit power for all the cases. The important observation from the figure is that the system throughput with the proposed SS intelligence is higher than in the conventional case without SS intelligence (up to $13 \%$ improvement for $R_{G}=100 \mathrm{~dB}$ ). Further, it can be
TABLE I. SimUlation PARAMETERS

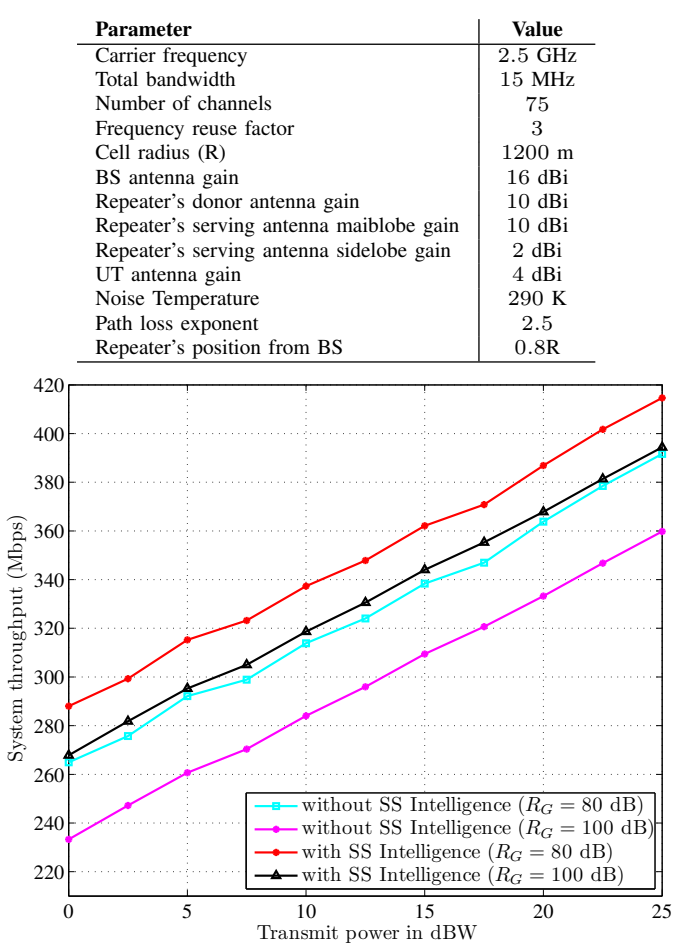

Fig. 2. System throughput versus BS transmit power

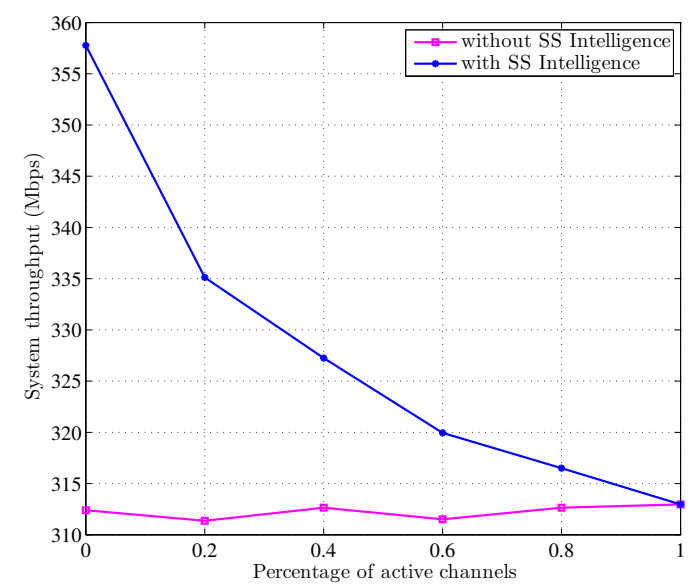

Fig. 3. System throughput versus percentage of the active channels $P_{t, B S}=$ $10 \mathrm{dBW}, R_{G}=100 \mathrm{~dB}$

depicted that the system throughput decreases with the increase in the repeater gain but higher performance gain with the SS intelligence is observed for the larger value of the repeater gain.

In order to analyze the effect of dynamic occupancy of the channels, we present the system throughput versus percentage of active channels in Fig. 3. From the figure, it can be depicted that the system throughput decreases with the increase in the percentage of active channels. This is due to the fact that as the number of active channels increases, the amplification bandwidth of the repeater increases and hence increasing the overall noise power at the UT. Another observation from the figure is that the rate of decrease is higher for the lower percentage of active channels than for the higher percentage of active channels. 
In practice, there may occur SS errors which may affect the total achievable capacity of the system. In this context, SS errors such as $P_{f}$ and $P_{m}$ need to be taken into account. In this context, in Fig. 4, we plot the system throughput achieved without considering SS error correction (measured throughput) and by considering SS error correction (effective throughput) as described in Section III-A. From the figure, it can be noted that the effective system throughput increases with the increase in the false alarm rate. This can be explained in the following way. When the value of $P_{f}$ increases, the measured number of active channels at the repeater increases and it increases the overall noise power at the UT. However, the actual number of active channels is small, hence less effective noise power and the higher effective system throughput.

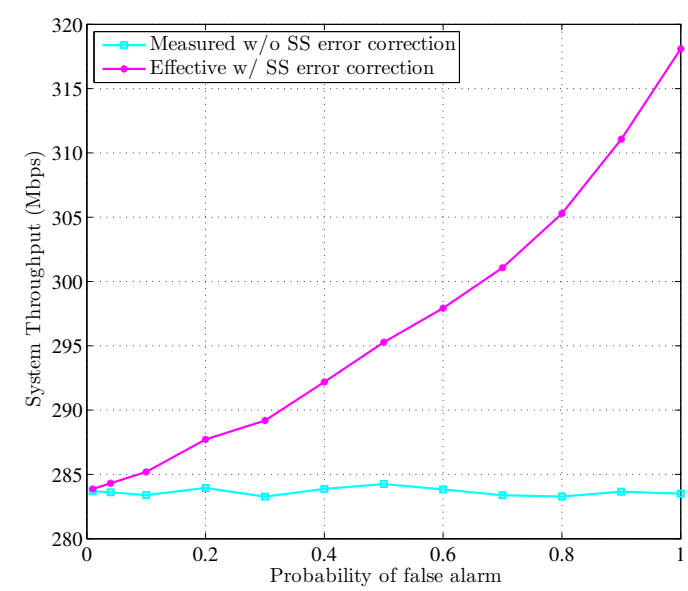

Fig. 4. System throughput versus probability of false alarm $\left(T_{s}=0.01 \mathrm{~s}\right.$, $\left.\gamma_{R}=5 \mathrm{~dB}, P_{t, B S}=10 \mathrm{dBW}, R_{G}=100 \mathrm{~dB}\right)$

\section{CONCLUSIONS AND FUTURE WORK}

The main shortcoming of an AF repeater is the amplification of the unnecessary noise and interference signals. In order to address this, a novel concept of an intelligent $5 \mathrm{G}$ AF repeater having SS intelligence has been presented and evaluated. Unlike conventional repeaters which tend to amplify the entire set of channels within a given assigned spectrum, the proposed repeater is capable of performing selective amplification of the active channels only. The performance of the proposed repeater was evaluated and compared with that of conventional repeaters. Results show that the capacity of a cellular system can be improved by replacing conventional repeaters with the proposed repeater. Simulation results demonstrated up to $13 \%$ throughput improvement for the considered set of parameters. Furthermore, it has been noted that the system capacity depends on the dynamic occupancy of the channels and SS errors which may occur during the sensing process. In our future work, we plan to study the proposed repeater from interference minimization perspective and to implement source localization and beamforming intelligence on the top of the proposed repeater in order to enhance the system capacity in SDMA-based 5G wireless systems.

\section{ACKNOWLEDGEMENT}

This work was partially supported by the National Research Fund, Luxembourg under the CORE project SeMIGod.

\section{REFERENCES}

[1] C.-X. Wang, and et al, "Cellular architecture and key technologies for 5G wireless communication networks," IEEE Commun. Mag., vol. 52, no. 2, pp. 122-130, Feb. 2014.

[2] M. Patwary, P. Rapajic, and I. Oppermann, "Capacity and coverage increase with repeaters in UMTS urban cellular mobile communication environment," IEEE Trans. Commun., vol. 53, no. 10, Oct. 2005.

[3] E. Drucker, "Development and application of a cellular repeater," in IEEE Veh. Technol. Conf., June 1988, pp. 321-325.

[4] Fijitsu, "Enhancing RF capacity via over-theair repeaters," whitepaper, Available online: http://www.fujitsu.com/downloads/TEL/fnc/whitepapers.

[5] P. Lahdekorpi, and et al, "Implementation aspects of RF-repeaters in cellular networks," in IEEE PIMRC, Sept 2010, pp. 2511-2516.

[6] D. Li, "Cognitive relay networks: Opportunistic or uncoded decodeand-forward relaying ?" IEEE Trans. Veh. Technol., vol. 63, no. 3, pp. 1486-1491, Mar. 2014.

[7] J. Liu, R. Love, and A. Nimbalker, "Recent results on relaying for LTE-advanced," in IEEE VTC-Fall, Sept. 2009, pp. 1-3.

[8] S. Sengupta and K. Subbalakshmi, "Open research issues in multi-hop cognitive radio networks," IEEE Commun. Mag., vol. 51, no. 4, pp. 168-176, Apr. 2013.

[9] Y. Xue, "Amplify-and-forward repeater enhanced random access in single-cell wireless communications," in IEEE PIMRC, Sept. 2008.

[10] H. Kha, and et al, "Joint optimization of source power allocation and cooperative beamforming for SC-FDMA multi-user multi-relay networks," IEEE Trans. Commun., vol. 61, no. 6, June 2013.

[11] S. K. Sharma, S. Chatzinotas, and B. Ottersten, "Spectrum sensing in dual polarized fading channels for cognitive SatComs," in IEEE Globecom Conf., Dec. 2012, pp. 3419 -3424.

[12] A. Goldsmith, S. Jafar, I. Maric, and S. Srinivasa, "Breaking spectrum gridlock with cognitive radios: An information theoretic perspective," Proc. IEEE, vol. 97, no. 5, pp. 894-914, May 2009.

[13] S. K. Sharma, S. Chatzinotas, and B. Ottersten, "SNR estimation for multi-dimensional cognitive receiver under correlated channel/noise," IEEE Trans. Wireless Commun., vol. 12, no. 12, Dec. 2013.

[14] S. K. Sharma, S. Chatzinotas, and B. Ottersten, "Compressive sparsity order estimation for wideband cognitive radio receiver," IEEE Trans. Signal Process., vol. 62, no. 19, Oct. 2014.

[15] M. Xiaomao and Q. Peiliang, "Cognitive relay," in Future Generation Commun. and Networking, vol. 2, Dec 2007, pp. 264-269.

[16] J. Lee, H. Wang, J. Andrews, and D. Hong, "Outage probability of cognitive relay networks with interference constraints," IEEE trans. Wireless Commun., vol. 10, no. 2, pp. 390-395, Feb. 2011.

[17] D. Wieruch, and et al, "Cognitive repeaters for flexible mobile data traffic offloading," in Int. Conf. CROWNCOM, July 2013, pp. 214-219.

[18] A. Kaushik, M. Raza, and F. Jondral, "On the deployment of cognitive relay as underlay systems," in Int. Conf. CROWNCOM, June 2014.

[19] H. Cheng and Y.-D. Yao, "Cognitive-relay-based intercell interference cancellation in cellular systems," IEEE Trans. Veh. Technol., vol. 59, no. 4, pp. 1901-1909, May 2010.

[20] W. C. Y. Lee and D. Lee, "The impact of repeaters on CDMA system performance," in IEEE VTC-Spring, vol. 3, 2000, pp. 1763-1767.

[21] S. K. Sharma, S. Chatzinotas, and B. Ottersten, "Eigenvalue-based sensing and SNR estimation for cognitive radio in presence of noise correlation," IEEE Trans. Veh. Technol., vol. 62, no. 8, Oct 2013.

[22] K. Patil, R. Prasad, and K. Skouby, "A survey of worldwide spectrum occupancy measurement campaigns for cognitive radio," in Int. Conf. Devices and Commun., Feb. 2011, pp. 1 -5.

[23] X. Song, and et al, "A statistical geometric approach for capacity analysis in two-hop relay communications," in IEEE Globecom, pp. 4823-4829, Dec. 2013

[24] S. K. Sharma, S. Chatzinotas, and B. Ottersten, "Exploiting polarization for spectrum sensing in cognitive SatComs," in 7th Int. Conf. CROWNCOM, June 2012, pp. $36-41$.

[25] Y.-C. Liang, Y. Zeng, E. Peh, and A. T. Hoang, "Sensing-throughput tradeoff for cognitive radio networks," IEEE Trans. Wireless Commun., vol. 7, no. 4, pp. 1326-1337, April 2008. 